\title{
Predictors of Death in Under-Five Children with Sepsis Attending an Urban Diarrheal Treatment Centre in Bangladesh
}

\author{
K. M. Shahunja ${ }^{1}$, Abu Sadat Mohammad Sayeem Bin Shahid ${ }^{1}$, Hasan Ashraf ${ }^{1,2}$, \\ Abu Syeed Golam Faruque ${ }^{1}$, Sumon Kumar Das ${ }^{1}$, Md. Kamruzzaman ${ }^{3}$, \\ Farhana Pervin Flora ${ }^{1}$, Mohammod Jobayer Chisti ${ }^{1,2^{*}}$
}

${ }^{1}$ Centre for Nutrition and Food Security, International Centre for Diarrhoeal Disease Research (icddr,b), Dhaka, Bangladesh; ${ }^{2}$ Clinical Service, Dhaka Hospital, International Centre for Diarrhoeal Disease Research (icddr,b), Dhaka, Bangladesh; ${ }^{3}$ Dhaka Shishu (Children) Hospital, Dhaka, Bangladesh.

Email: *chisti@icddrb.org

Received May $10^{\text {th }}, 2013$; revised June $10^{\text {th }}, 2013$; accepted June $18^{\text {th }}, 2013$

Copyright (C) 2013 K. M. Shahunja et al. This is an open access article distributed under the Creative Commons Attribution License, which permits unrestricted use, distribution, and reproduction in any medium, provided the original work is properly cited.

\begin{abstract}
SETTING: Special Care Ward (SCW) of the Dhaka Hospital of icddr,b, Bangladesh. OBJECTIVE: To evaluate the clinical and laboratory predictors of death in under-five children with clinically defined sepsis presenting with diarrhea. METHODS: We prospectively enrolled all the diarrheal children $(\mathrm{n}=151)$ aged 0 to 59 months with clinical sepsis admitted in the SCW during September'2007 through December'2007. Comparison was made between deaths $(\mathrm{n}=23)$ and survivors $(\mathrm{n}=128)$. Sepsis is defined as presence of inflammation [abnormal WBC count $\left(>11 \times 10^{9} / \mathrm{L}\right.$ or, $<4 \times$ $10^{9} / \mathrm{L}$ or, band and neutrophil ratio $\left.\leq 0.10\right]$ plus presence or presumed presence of infection with thermo-instability [hypo $\left(\leq 35.0^{\circ} \mathrm{C}\right)$ or hyperthermia $\left(\geq 38.5^{\circ} \mathrm{C}\right)$ ], tachycardia, tachypnea, and/or the indications of altered organ function (altered mental status and bounding pulse) in the absence of clinical dehydration or after correction of dehydration. RESULTS: The median (inter-quartile range) age (months) of the children who survived and died was comparable [4.0 $(2.0,12.0)$ vs. $1.5(0.8,10.0) ; \mathrm{p}=0.703]$. In the logistic regression analysis, after adjusting for potential confounders, such as abnormal WBC count, use of intravenous fluid, patient with fatal outcome more often presented with hypernatremia (odds ratio $=16.48,95 \%$ confidence interval $=2.21-123.12 ; p=0.006)$, lobar consolidation $($ odds ratio $=19.9$, $95 \%$ confidence interval $=2.99-132.80 ; \mathrm{p}=0.002)$, hypoxemia $($ odds ratio $=14.78,95 \%$ confidence interval $=1.38$ $157.90 ; \mathrm{p}=0.026$ ) and severe under-nutrition (odds ratio $=7.57,95 \%$ confidence interval $=1.24-46.11 ; \mathrm{p}=0.028$ ). CONCLUSIONS: Our data suggest that children under-five with clinical sepsis who present with lobar pneumonia, hypoxaemia, severe acute malnutrition and hyperntaemia are at higher risk of death and identification of these simple factors may help clinicians to take prompt initiative for the aggressive management of such children especially in a resource-limited setting like Bangladesh.
\end{abstract}

Keywords: Bangladesh; Diarrhea; Hypoxemia; Sepsis; Severe Malnutrition

\section{Introduction}

Sepsis is one of the most important causes of death in under-five children in developing countries $[1,2]$. The largest part of the global sepsis burden occurs in middleand low-income countries. Around $70 \%$ of the 7.6 million global deaths in neonates and infants are attributable to sepsis, with the majority of cases occurring in Asia and sub-Saharan Africa [3]. There are between 77 to 240 new cases of sepsis per 100,000 population each year

${ }^{*}$ Corresponding and senior author.
$[4,5]$ and the incidence may continue to increase by approximately $1.5 \%$ in every year, resulting in an additional 1 million cases per year by $2020[4,6,7]$. As sepsis is the final common pathway in most of the pediatric diseases and presence of sepsis in children with diarrhea is often associated with high case-fatality [8], sepsis may be considered the number one killer of children worldwide [9]. Thus, clinicians as well as health workers in resource limited settings should have understanding on simple associated factors that may predict fatal outcome 
in diarrheal children with sepsis. However, data are very limited on the predicting factors of death in such population.

In the Dhaka Hospital of icddr,b, a number of children used to be admitted on a daily basis with diarrheal diseases along with other co-morbidities such as severe acute malnutrition, severe and very severe pneumonia, with the consequence of sepsis, and often with fatal outcome. The aim of our study was to identify the factors that predict deaths in under-five children with sepsis admitted to urban diarrheal treatment centre.

\section{Materials and Methods}

\subsection{Patient Enrollment}

All the diarrheal children of either sex, aged 0 - 59 months, with clinical sepsis who required admission and care to the Special Care Ward (SCW) of the Dhaka Hospital of icddr,b from September, 2007 to December, 2007 were enrolled in this study. The hospital provides care and treatment to around 110,000 patients of all ages each year. The majority of the patients is residents of Dhaka city or its suburbs and come from poor socio-economic backgrounds. Nearly all patients attend the hospital with diarrheal diseases, whether associated with complications or other associated health problems. On arrival to the hospital triage, nurses obtain the medical history and perform a rapid assessment of the patients, focusing on the nature and severity of diarrhea, including the magnitude of dehydration and any complications. Features of any other health problems are also sought at presentation. Following triage, patients are also examined and assess by the attending physician for consultation or admission for an appropriate ward of the hospital. Patients with severe illnesses, including those with altered mental status, convulsions, severe or very severe pneumonia, hypoxemia, cyanosis and suspected sepsis, are admitted directly to the SCW. On admission at SCW the attending physician again perform a thorough assessment of the patients, organize any investigation necessary and determine the management plan including drug and other supportive therapies. Our study patients were admitted to SCW having diarrhea and sepsis with or without other associated problems such as electrolytes imbalance, severe acute malnutrition, features of severe/very severe pneumonia and hypoxemia.

\subsection{Study Design}

In this prospective cohort study, comparison of the clinical and laboratory features of under-five diarrheal children with sepsis was made who had and who did not have fatal outcome. Consent was obtained from respective parents or the attending family members before chil- dren were enrolled in the study and relevant clinical information were collected. Clinical management of all children for the sepsis was done according to the standard management guidelines of the hospital based on the surviving sepsis guideline [10]. These include management by restoration of circulation, respiration, maintenance of temperature and blood glucose level, appropriate antimicrobial therapy to cover the possible causative organism, appropriate feeding; and provision of micronutrients, viatamins and minerals as required. The hospital follows the protocolized guidelines for the management of children with severe protein-energy malnutrition $[11,12]$.

\subsection{Recognizing the Septic Patient}

\subsubsection{Definition}

\section{Sepsis}

Sepsis is defined as presence of inflammation [abnormal WBC count $\left(>11 \times 10^{9} / \mathrm{L}\right.$ or, $<4 \times 10^{9} / \mathrm{L}$ or, band and neutrophil ratio $\leq 0.10$ ) plus presence or presumed presence of infection with thermo-instability [hypothermia ( $\leq$ $\left.35.0^{\circ} \mathrm{C}\right)$ or hyperthermia $\left.\left(\geq 38.5^{\circ} \mathrm{C}\right)\right]$, tachycardia, tachypnea, and/or the indications of altered organ function (altered mental status and bounding pulse) in the absence of clinical dehydration or after correction of dehydration [1].

\subsubsection{Statistical Methods}

We used pretested case report forms (CRF) for data acquisition. All CRFs were manually verified for completeness and errors, edited where necessary, and then data entered onto a personal computer (PC). Data were entered and analyzed using SPSS for Windows (version 17.0; SPSS Inc, Chicago, IL, USA) and Epi Info (version 6.0; USD, Stone Mountain, GA, USA). The differences in proportions were compared by the chi-square test and differences of means by Student's t-test or Mann-Whitney test, as appropriate. A probability of $<0.05$ was considered statistically significant.

Strength of association was determined by estimating the odds ratio (OR) and the $95 \%$ confidence intervals (CI). Univariate analysis and logistic regression were both performed in identifying risk factors. The analyses of socio-demographic data included age, sex, gestational age, residence (in Dhaka or not), maternal and paternal education, mothers' occupation, breastfeeding status, not use high-potency vitamin A capsule within 6 months prior to admission, and use of antibiotics before admission. The analyses of clinical data included presence or absence of dehydration, cyanosis, hypoxemia, fever, hypothermia, severe malnutrition and requirement of IV fluid after admission. The laboratory data analyses included total peripheral white blood cell (WBC) count, presence of immature neutrophils in blood such as band, 
serum electrolytes, blood glucose, radiological pneumonia. Initially, univariate analyses of these factors were performed to evaluate their association with fatal outcome, and then logistic regression analysis was performed to identify the independent predictors of death where death was the dependent variable and the factors that were significantly associated with deaths in the univariate analysis acted as independent variables.

\section{Results}

Among the 151 under-five diarrheal children with sepsis admitted to the SCW of the Dhaka hospital of icddr,b 23 $(15 \%)$ had fatal outcome. In univariate analyses, children who died more often had hypoxemia, severe under-nutrition, higher total leucocyte count, and lobar consolidation compared to those who survived (Tables 1 and 2). Proportionately higher children with fatal outcome presented with edematous malnutrition and immature poly on admission compared to those who survived, but it was not statistically significant (Tables 1 and 2). In logistic regression analysis, after adjusting for potential confounders such as use of $\mathrm{I} / \mathrm{V}$ fluid after admission and total WBC count, severe under nutrition, hypoxemia, lobar consolidation and hypernatremia were remained as independent predictors of death in sepsis (Table 3). Other parameters in Tables 1 and 2 were comparable among the groups.

Table 1. Clinical characteristics of the under-five children with clinical sepsis who died (cases) and survived (controls).

\begin{tabular}{|c|c|c|c|c|c|}
\hline Variables & Deaths $(n=23)$ & Survivors $(n=128)$ & OR & $95 \% \mathrm{CI}$ & $\mathrm{p}$ \\
\hline Male gender & $13(57)$ & $75(59)$ & 0.92 & $0.34-2.46$ & 0.865 \\
\hline Age in months (Median, IQR) & $1.5(0.8,10.0)$ & $4.0(2.0,12.0)$ & - & - & 0.703 \\
\hline Gestational age (weeks) (mean \pm standard deviation) & $38.0 \pm 2.9$ & $38.0 \pm 3.7$ & - & - & 0.974 \\
\hline Not use of capsule vitamin-A within last 6 months & $11(50)$ & $72(56)$ & 0.78 & $0.29-2.10$ & 0.755 \\
\hline History of measles within last 6 months & $2(9)$ & $20(16)$ & 0.52 & $0.08-2.59$ & 0.530 \\
\hline Use of antibiotic before admission & $13(59)$ & $52(43)$ & 1.92 & $0.70-5.32$ & 0.245 \\
\hline Clinical dehydration (some/severe) & $15(65)$ & $68(53)$ & 1.65 & $0.60-4.62$ & 0.398 \\
\hline Cyanosis & $4(17)$ & $14(11)$ & 1.71 & $0.42-6.4$ & 0.481 \\
\hline Fever $\left(\geq 38^{\circ} \mathrm{C}\right)$ & $19(83)$ & $111(87)$ & 0.73 & $0.20-2.88$ & 0.530 \\
\hline Hypothermia on or after admission $\left(\mathrm{Temp} \leq 35^{\circ} \mathrm{C}\right)$ & $2(9)$ & $5(4)$ & 2.34 & $0.29-15.08$ & 0.289 \\
\hline Hypoxaemia $\left(\mathrm{SpO}_{2}<90 \%\right)$ & $19(83)$ & $52(41)$ & 6.94 & $2.06-25.73$ & $<0.001$ \\
\hline Oedematous malnutrition & $5(22)$ & $10(8)$ & 3.28 & $0.86-12.16$ & 0.055 \\
\hline HAZ $(<-3 \mathrm{z}$ score $)$ & $10(44)$ & $38(30)$ & 1.82 & $0.67-4.93$ & 0.287 \\
\hline WAZ $(<-3$ z score $)$ & $16(70)$ & $48(38)$ & 3.81 & $1.35-11.10$ & 0.008 \\
\hline WHZ $(<-3$ z score $)$ & $6(26)$ & $35(27)$ & 0.94 & $0.30-2.80$ & 0.897 \\
\hline User of IV fluid & $17(74)$ & $75(59)$ & 2.00 & $0.68-6.13$ & 0.248 \\
\hline
\end{tabular}

Figures represent $\mathrm{n}(\%)$, unless specified. OR: odds ratio; CI: confidence interval; IQR: inter-quartile range; HAZ: height for age $\mathrm{z}$ score; WAZ: weight for age $\mathrm{z}$ score; WHZ: weight for height $\mathrm{z}$ score; $\mathrm{SpO}_{2}=$ transcutaneously measured blood oxygen concentration.

Table 2. Laboratory characteristics of the under-five children with clinical sepsis who died (cases) and survived (controls).

\begin{tabular}{|c|c|c|c|}
\hline Variables & Cases $(n=23)$ & Controls $(\mathrm{n}=128)$ & $\mathrm{p}$ \\
\hline $\begin{array}{c}\text { Total WBC count (number/cu. mm) } \\
\text { (Median, IQR) }\end{array}$ & $11,000(8900,25,350)$ & $14,500(9300,20,000)$ & 0.046 \\
\hline Immature Poly (number/cu. mm) (Median, IQR) & $00(00,1.00)$ & $00(00,00)$ & 0.087 \\
\hline Hyponatraemia $(\mathrm{mmol} / \mathrm{L})$ & $5(22)$ & $33(26)$ & $\begin{array}{c}0.880 \\
0.80(0.24-2.54)^{*}\end{array}$ \\
\hline Hypernatraemia $(\mathrm{mmol} / \mathrm{L})$ & $5(22)$ & $11(9)$ & $\begin{array}{c}0.072 \\
2.95(0.78-10.74)^{*}\end{array}$ \\
\hline Hypokalaemia $(\mathrm{mmol} / \mathrm{L})$ & $8(35)$ & $34(27)$ & $\begin{array}{c}0.577 \\
1.47(0.52-4.13)^{*}\end{array}$ \\
\hline Hyperkalaemia $(\mathrm{mmol} / \mathrm{L})$ & $3(13)$ & $9(7)$ & $\begin{array}{c}0.395 \\
1.98(0.39-9.05)^{*}\end{array}$ \\
\hline Lobar consolidation & $9(56)$ & $6(7)$ & $\begin{array}{c}<0.001 \\
16.93(3.99-76.96)^{*}\end{array}$ \\
\hline Hypoglycemia $(\mathrm{RBS}<3 \mathrm{mmol} / \mathrm{L})$ & $3(13)$ & $17(14)$ & $\begin{array}{c}1.00 \\
0.96(0.20-3.96)^{*}\end{array}$ \\
\hline Growth on blood culture & $1 / 15(7)$ & $12 / 111(11)$ & $\begin{array}{c}1.00 \\
0.57(0.03-4.86)^{*}\end{array}$ \\
\hline
\end{tabular}

Figures represent n (\%), unless specified. IQR: interquartile range; "OR (95\% CI); RBS: Random blood sugar. 
Table 3. Results of logistic regression to explore the independent predictors of death in under-five children with sepsis.

\begin{tabular}{cccc}
\hline Characteristics & OR & 95\% CI & P \\
\hline Severe under nutrition & 7.57 & $1.24-46.11$ & 0.028 \\
Hypoxemia & 14.78 & $1.38-157.90$ & 0.026 \\
Lobar consolidation & 19.9 & $2.99-132.80$ & 0.002 \\
Hypernatraemia & 16.48 & $2.21-123.12$ & 0.006 \\
Total WBC count & 1.00 & $1.00-1.00$ & 0.156 \\
User of IV fluid after admission & 0.54 & $0.09-3.08$ \\
\hline
\end{tabular}

\section{Discussion}

Our finding of the association of severe malnutrition, lobar pneumonia and hypoxemia as predictors of death in SCW is similar to earlier reports $[13,14]$. Severely malnourished children have depressed cell mediated as well as humoral immune responses, and they are more susceptible to infection often with serious ramification such as fatal outcome [15].

We observed that lobar consolidation in septic children was one of the predictors of death. Lobar consolidation is usually associated with bacterial pneumonia which is often associated with fatal outcome especially in children under five in developing countries like Bangladesh [13], mostly due to overwhelming hypoxemia resulting from ventilation perfusion mismatch from para-pneumonic shunting. [14,16,17]. Sepsis may be the consequences of lobar pneumonia, as observed in our cases, frequently associated with high case-fatality rate [14].

Our observation of hypernatraemia as a predictor of death is also understandable. Hypernatraemic dehydration is usually associated with high case fatality either because of intracranial hemorrhage resulting from intracellular dehydration or because of cerebral oedema as a result of aggressive correction [18]. The observation of high case fatality in children with hypernatraemia has been reported earlier from Bangladesh [19]. The management of hypernatremic dehydration in diarrheal children remains a challenge, and more works need to be carried out into the treatment of this condition.

We observed that a higher proportion of children with fatal outcome had a leucocytosis and immature neutrophils. Increased number of immature neutrophils in the peripheral blood smears of children is an expression of severe illness common in bacterial sepsis [1]. An earlier study from Bangladesh reported that children with leukocytosis and immature neutrophils in their peripheral blood are more likely to be bacteremic and to have a higher risk of death [1]. Thus, the presence of immature neutrophils in peripheral blood in diarrheal children should alert clinicians to look for sepsis in order to reduce morbidity and deaths in such population.

The number of children with fatal outcome in our study was much smaller compared with the control children. This might have reduced the power to identify fac- tors that could actually have an independent association with fatal outcome. Further studies using adequate number of cases could provide better insight.

In conclusion, our data suggest that under-five diarrheal children with clinical sepsis admitted to a special care ward of a resource-limited setting in a developing country, who present with severe malnutrition, hypernatraemia, lobar pneumonia and hypoxaemia are more likely to have a fatal outcome. Thus, efforts should be taken to specifically look for the presence of these features in all hospitalized diarrheal children with clinical sepsis to identify those with higher risk of death for the institution of energetic and efficient antimicrobial and other supportive therapy with the prospect of achieving a better outcome.

\section{Acknowledgements}

We gratefully acknowledge the donors for their support and commitment to icddr,b's research efforts which currently provide unrestricted support to the centre's research efforts: Government of the People's Republic of Bangladesh, Canadian International Development Agency (CIDA), Embassy of the Kingdom of the Netherlands (EKN), Swedish International Development Cooperation Agency (Sida) and the Department for International Development, UK (DFID). We offer our sincere gratitude to all physicians including clinical fellows, nurses, members of the feeding team and cleaners of SCW for their invaluable contribution during patient enrollment and data collection.

\section{REFERENCES}

[1] M. J. Chisti , S. Saha, C. N. Roy and M. A. Salam, "Predictors of Bacteremia in Infants with Diarrhea and Systemic Inflammatory Response Syndrome Attending an Urban Diarrheal Treatment Center in a Developing Country," Pediatric Critical Care Medicine, Vol. 11, No. 1, 2010, pp. 92-97. doi:10.1097/PCC.0b013e3181b063e1

[2] A. C. Cheng, T. E. West, D. Limmathurotsakul and S. J. Peacock, "Strategies to Reduce Mortality from Bacterial Sepsis in Adults in Developing Countries," PLOS Medicine, Vol. 5, No. 8, 2008, p. e175. doi:10.1371/journal.pmed.0050175

[3] L. Liu, H. L. Johnson, S. Cousens, J. Perin, S. Scott and 
J. E. Lawn, "Global, Regional, and National Causes of Child Mortality: An Updated Systematic Analysis for 2010 with Time Trends since 2000," Lancet, Vol. 379, No. 9832, 2012, pp. 2151-2161. doi:10.1016/S0140-6736(12)60560-1

[4] G. S. Martin, D. M. Mannino, S. Eaton and M. Moss, "The Epidemiology of Sepsis in the United States from 1979 through 2000," New England Journal of Medicine, Vol. 348, No. 16, 2003, pp. 1546-1554.

[5] S. Finfer, R. Bellomo, J. Lipman, C. French, G. Dobb and J. Myburgh, "Adult-Population Incidence of Severe Sepsis in Australian and New Zealand Intensive Care Units," Intensive Care Medicine, Vol. 30, No. 4, 2004, pp. 589596. doi:10.1007/s00134-004-2157-0

[6] D. C. Angus, W. T. Linde-Zwirble, J. Lidicker, G. Clermont, J. Carcillo and M. R. Pinsky, "Epidemiology of Severe Sepsis in the United States: Analysis of Incidence, Outcome, and Associated Costs of Care," Critical Care Medicine, Vol. 29, No. 7, 2001, pp. 1303-1310. doi:10.1097/00003246-200107000-00002

[7] V. Y. Dombrovskiy, A. A. Martin, J. Sunderram and H. L. $\mathrm{Paz}$, "Rapid Increase in Hospitalization and Mortality Rates for Severe Sepsis in the United States: A Trend Analysis from 1993 to 2003," Critical Care Medicine, Vol. 35, No. 5, 2007, pp. 1244-1250. doi:10.1097/01.CCM.0000261890.41311.E9

[8] M. J. Chisti, T. Duke, C. F. Robertson, T. Ahmed, A. S. G. Faruque and P. K. Bardhan. "Co-Morbidity: Exploring the Clinical Overlap between Pneumonia and Diarrhoea in a Hospital in Dhaka, Bangladesh," Annals of Tropical Paediatrics, Vol. 31, No. 4, 2011, pp. 311-319. doi:10.1179/1465328111Y.0000000033

[9] C. Riley and D. S. Wheeler. "Prevention of Sepsis in Children: A New Paradigm for Public Policy," Critical Care Research and Practice, Vol. 2012, No. 437139, 2012, p. 8. doi: $10.1155 / 2012 / 437139$

[10] R. P. Dellinger, M. M. Levy, A. Rhodes, D. Annane, H. Gerlach, S. M. Opal, J. E. Sevransky, C. L. Sprung, I. S. Douglas, R. Jaeschke, T. M. Osborn, M. E. Nunnally, S. R. Townsend, K. Reinhart, R. M. Kleinpell, D. C. Angus, C. S. Deutschman, F. R. Machado, G. D. Rubenfeld, S. A. Webb, R. J. Beale, J. L. Vincent and R. Moreno, "Surviving Sepsis Campaign Guidelines Committee including the Pediatric Subgroup. Surviving Sepsis Campaign: International Guidelines for Management of Severe Sepsis and Septic Shock," Critical Care Medicine, Vol. 41, No. 2, 2013, pp. 580-637. doi:10.1097/CCM.0b013e31827e83af
[11] T. Ahmed, M. Ali, M. M. Ullah, I. A. Choudhury, M. E. Haque and M. A. Salam, "Mortality in Severely Malnourished Children with Diarrhea and Use of a Standardized Management Protocol," Lancet, Vol. 353, No. 9168, 1999, pp. 1919-1922.

[12] WHO, "WHO Management of Severe Malnutrition: A Manual for Physicians and other Senior Health Workers," World Health Organization, Geneva, 1999.

[13] M. J. Chisti, T. Ahmed, A. S. Faruque and M. A. Salam, "Clinical and Laboratory Features of Radiologic Pneumonia in Severely Malnourished Infants Attending an Urban Diarrhea Treatment Center in Bangladesh," Pediatric Infectious Disease Journal, Vol. 29, No. 2, 2010, pp. 174-177. doi:10.1097/INF.0b013e3181b9a4d5

[14] M. J. Chisti, M. Tebruegge, S. L. Vincente, S. M. Graham and T. Duke, "Pneumonia in Severely Malnourished Children in Developing Countries-Mortality Risk, Aetiology and Validity of WHO Clinical Signs: A Systematic review," Tropical Medicine \& International Health, Vol. 14, No. 10, 2009, pp. 1173-1179. doi:10.1111/j.1365-3156.2009.02364.x

[15] G. Morgan, "What, If Any, Is the Effect of Malnutrition on Immunological Competence?" Lancet, Vol. 349, No. 9066, 1997, pp. 1693-1695. doi:10.1016/S0140-6736(96)12038-9

[16] L. Liu, Q. Li, R. A. Lee, I. K. Friberg, J. Perin, N. Walker and R. E. Black, "Trends in Causes of Death among Children under 5 in Bangladesh, 1993-2004: An Exercise Applying a Standardized Computer Algorithm to Assign Causes of Death Using Verbal Autopsy Data," Population Health Metrics, Vol. 9, 2011, p. 43. doi:10.1186/1478-7954-9-43

[17] M. W. Weber, S. Usen, A. Palmer, S. Jaffar and E. K. Mulholland, "Predictors of Hypoxaemia in Hospital Admissions with Acute Lower Respiratory Tract Infection in a Developing Country," Archives of Disease in Childhood, Vol. 76, No. 4, 1997, pp. 310-314. doi:10.1136/adc.76.4.310

[18] H. J. Adrogue and N. E. Madias, "Hypernatremia," New England Journal of Medicine, Vol. 342, No. 20, 2000, pp. 1493-1499. doi:10.1056/NEJM200005183422006

[19] A. R. Sadmani , M. A. Wahed, M. R. Islam and S. M. Ahmed, "Consequence of Hypernatraemia and Hypernatraemia in Children with Acute Diarrhoea in Bangladesh," British Medical Journal (Clinical Research Ed), Vol. 286, No. 6366, 1983, pp. 671-673. doi: $10.1136 / \mathrm{bmj} .286 .6366 .671$ 


\section{Elaboration of Abbreviations:}

CI: Confidence interval

CIDA: Canadian International Development Agency

CRF: Case report form

EKN: Embassy of the Kingdom of the Netherlands

HAZ: Height for age $z$ score

IQR: Inter-quartile range

IV: Intra-venous

mmol/L: Milimol/Liter

OR: Odds ratio
RBS: Random blood sugar

SCW: Special care ward

Sida: Swedish International Development Cooperation Agency

$\mathrm{SpO}_{2}$ : Transcutaneously measured blood oxygen concentration

DFID: Department for International Development

WAZ: Weight for age $\mathrm{z}$ score

WBC: White blood count

WHZ: Weight for height/length z score 